\title{
Modeling the performance of distributed fiber optical sensor based on spontaneous Brillouin scattering
}

Mei, Ying, Xu, Xiaomin, Soga, Kenichi

Ying Mei, Xiaomin Xu, Kenichi Soga, "Modeling the performance of distributed fiber optical sensor based on spontaneous Brillouin scattering," Proc. SPIE 11340, AOPC 2019: Optical Fiber Sensors and Communication, 1134010 (18 December 2019); doi: 10.1117/12.2548121

SPIE. Event: Applied Optics and Photonics China (AOPC2019), 2019, Beijing, China 


\title{
Modeling the performance of distributed fiber optical sensor based on spontaneous Brillouin scattering
}

\author{
Ying Mei* ${ }^{\text {ab }}$, Xiaomin $\mathrm{Xu}^{\mathrm{b}}$, Soga Kenichi ${ }^{\mathrm{bc}}$ \\ ${ }^{a}$ Beijing Institute of Aerospace Control Devices, Beijing Engineering Research Center of Optical \\ Fiber Sensing System, No.1 Fengying East Road, Haidian District, Beijing, China, 100094; \\ ${ }^{b}$ Department of Engineering, University of Cambridge, Cambridge, CB2 1PZ, UK; \\ ${ }^{c}$ Department of Civil and Environmental Engineering, University of California at Berkeley, \\ Berkeley, CA 94720 USA
}

\begin{abstract}
An optical model to simulate the distributed fiber optical sensor based on spontaneous Brillouin spectrum is derived. The reliability of this model is validated with experimental measurements. Using this analytical expression, parametric studies are conducted to investigate impacts of key factors including fiber loss, signal to noise ratio, bandwidth and scanning step on the optical fiber sensor measurement error. The simulation results exhibit good agreement with previous published calculation results. Applying this novel model into the data interpretation, measurement error of distributed fiber optical sensor based on spontaneous Brillouin scattering can be better controlled.
\end{abstract}

Keywords: spontaneous Brillouin scattering, distributed fiber optical sensor, analytical model, strain measurement

\section{INTRODUCTION}

Spontaneous Brillouin scattering is the scattering of light from sound waves. It is one of the basic scattering mechanisms of the distributed sensing techniques, which commonly occur in single mode optical fibers. In theory, spontaneous Brillouin scattering consists of the coupling between the injected pulsed wave and the vibration modes of the fiber itself, which are sound waves. The backscattered Brillouin wave has a different peak frequency than the injected pulse. The frequency shift is dependent on the temperature and strain conditions of the cable. Based on this, the distributed fiber optics sensor measures the backscattered Brillouin gain spectrum as a function of the frequency shift to resolve the strain/temperature condition of the cable[1].

To compensate for measurement error and achieve reliable interpretation of the measured strain profile, it is necessary to establish a model to connect analyzer measurements to real strain/temperature conditions based on Brillouin sensing mechanism. Fundamental models have been developed in the past to simulate the process of Brillouin scattering on the cable[2][3]. One approach to simulate the backscattered Brillouin power is to only consider the interaction between the pulsed light and the backscattered Stokes wave[4][5]. With some assumptions, such as expressing the measured Brillouin profile as a convolution of the injected pulse light and the intrinsic Lorentz shape distribution, the model is simplified[6][7]. By considering the coupling of the three waves (the pump wave, Brillouin backscattered wave, and acoustic wave), the Brillouin backscattering model is improved[8][9]. This result indicates the analyzer measured backscattered spectrum can be expressed as a convolution of Brillouin spectrum with a spread function over time.

This paper presents an optics model for fundamentally simulating the Brillouin scattering process. The model exploits a weight function to simulate the convolution process of the analyzer. A cable with a step strain is tested with a Brillouin optical fiber sensor to validate the reliability of this model. The characteristics of the measurement error and their dependency factors are investigated using the model.

\section{THEORETICAL ANALYSIS}

In a BOTDR system, a pulsed light is injected into one end of an optical fiber, and the backscattered Brillouin light produced in the fiber is received and processed at the same end of the fiber. The power of the Brillouin backscattered light $P_{B}(z, v)$ which is produced in a small section of the fibre can be expressed using as[10]:

*Email:sophiamy686171@163.com; Tel: +86-13121829229 


$$
d P_{B}(z, v)=g_{B}\left(v, v_{B}\right)\left(\frac{c_{0}}{2 n}\right) P(z) d x \times \exp \left(-2 \alpha_{z} z\right)
$$

Where $P$ is the launched pulse power, $c_{0}$ is the light velocity in vacuum, $n$ is the fibre refractive index, $\alpha_{z}$ is the attenuation coefficient of the fibre, $z$ is the scattering position, and $g_{B}\left(v, v_{B}\right)$ is the Brilouin gain spectrum with a Lorentzian shape, $v_{B}$ is the centre frequency at which $g_{B}\left(v, v_{B}\right)$ has a peak power.

Assuming that the launched pulse is only amplitude-modulated and has a constant optical frequency in the frequency domain within the pulse duration, the input pulse is modelled as a square pulse. Figure 1 schematically illustrates an injected pulse which starts at time $t_{0}$, with a duration of $\tau$, rising and falling time $\Delta \tau$.

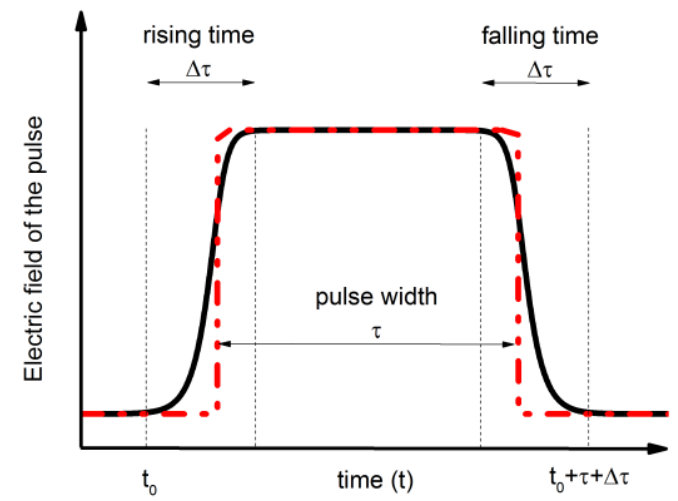

Figure 1. Graphically illustration of a squared pulse(black line) with rising/falling time of $\Delta \tau$ and a pulse width of $\tau$.

The power leakage of the pulse can be quantified by the extinction ratio which is the power ratio between the leak and the pulse power. With a known input peak power $\left(P_{P}\right)$ and an extinction ratio $(E R)$, the input leak power $\left(L_{p}\right)$ of the pulse can be calculated as $P_{P} \times 10^{-E R / 10}$. The electric field $E(t)$ of the launched pulse is then given by:

$$
E(t)=\left(\sqrt{P_{P}}-\sqrt{L_{P}}\right) \times \sqrt{\frac{\tanh \left(\frac{t-t_{0}}{0.45 \Delta \tau}\right)-\tanh \left(\frac{t-t_{0}-\tau}{0.45 \Delta \tau}\right)}{2}}+\sqrt{L_{P}}
$$

The pulsed light power spectrum in the frequency domain can be obtained by doing a Fourier transfer of the $E(t)$ :

$$
P_{P}(f)=F F T(E(t))
$$

The backscattered Brillouin spectrum is a convolution of the pulse and the intrinsic Lorentz distribution with key parameters dependent on the fibre strain/temperature condition. As shown in Fig.2, for a launched pulse with a center optical frequency of $f_{0}$, a small width $d f$ of the spectrum generates a Lorentzian shape backscattered Brillouin spectrum with a centre frequency of $f-S_{B}$. The Brillouin backscattered light power spectrum generated by the whole launched pulse is given by a Lorentzian profile with a peak power frequency of $f-S_{B}$ and a full width at half-maximum of $\Delta v$. 


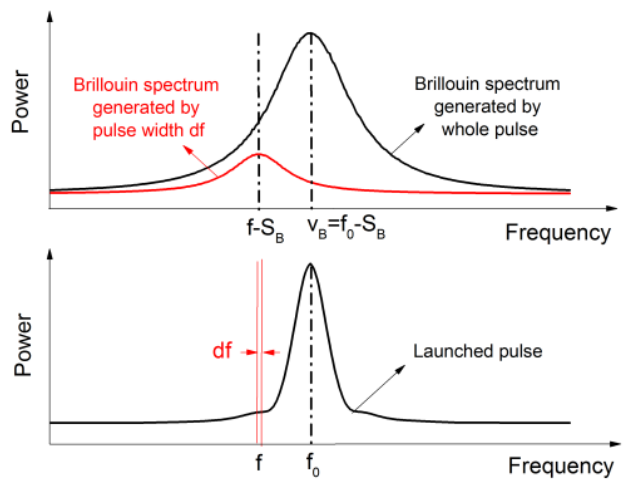

Figure 2. Generation of a Brillouin spectrum from a pulsed light

The obtained Brillouin backscattered spectrum is simulated as a weighted average spectrum over the analyzer's spatial resolution, which is an integration between the intrinsic spectrum and a weight function. The backscattered Brillouin spectrum profile $P_{B}(z, v)$ is obtained through integrating Eq. (1), and the measured Brillouin spectrum after analyzer processing can be expressed as:

$$
P_{M B}(z, v)=\int_{z-l / 2}^{z+L / 2} P_{B}(z, v) W(x) \alpha(z) d x
$$

in which $W(x)$ is a weight function with a shape dependent on the analyzer characteristics, and $\alpha(\mathrm{z})$ is the fibre refractive index.

\section{EXPERIMENTAL VERIFICATION}

The reliability of the model was firstly validated with laboratory tests where a section of $1.5 \mathrm{~m}$ reinforced strain sensing cable was fixed at one end and stretched at the other end. Measurements were taken at three different strain levels (around $1250 \mu \varepsilon, 2000 \mu \varepsilon$ and $2800 \mu \varepsilon$ ) by the Neubrex NBX-5000 BOTDR analyzer with the spatial resolution set as $1 \mathrm{~m}$.

Figure 3 shows the comparison of the laboratory local straining inputs, BOTDR measurement results and simulation results computed with the evaluated weight function which follows a normal distribution and a bandwidth of $0.185 \mathrm{~m}$. A good agreement between the simulation and experimental values can be clearly seen in the figure. The correlations between the simulation and experimental values are above $99 \%$ for all three straining cases. The error between the experiment results and the simulated curve is less than $11 \mu \varepsilon$. 


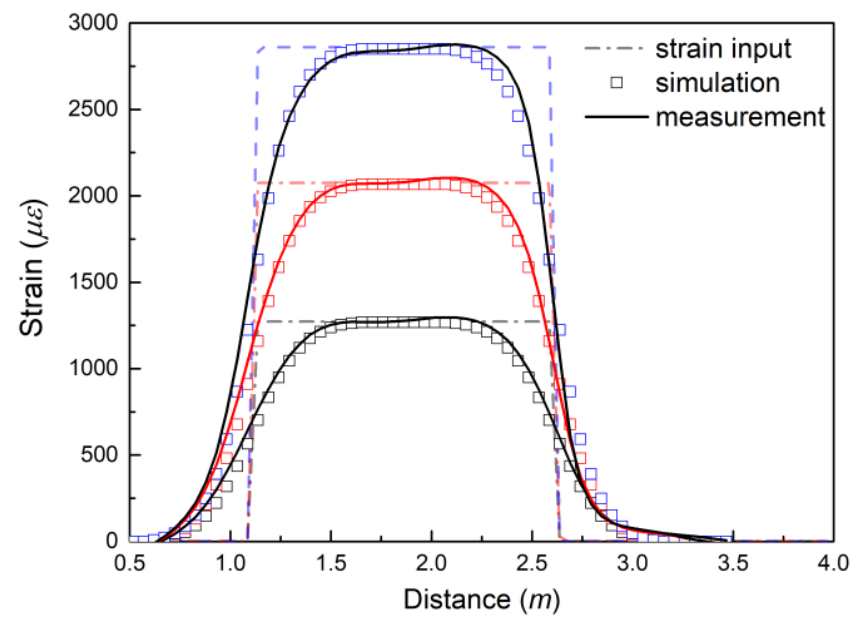

Figure 3. Comparison of the simulated results and the analyzer measured results at different strain input levels.

Figure 3 also implies that error is more significant at the strain change sections (distance $=0.75 \mathrm{~m}-1.25 \mathrm{~m}$ and $2.25 \mathrm{~m}$ $2.75 \mathrm{~m}$ ). For a BOTDR analyzer with a certain spatial resolution(one meter in this case), a non-uniformly distributed strain over spatial resolution will distort the received Brillouin spectrum or induce additional peaks to the spectrum. As a result, strain measurement will have a larger error at points where strain changes rapidly (i.e. a step function in this case).

The reliability of the optics model in describing strain in a length shorter than the analyzer's spatial resolution was then validated. The strain sensing cable was tensioned at $1200 \mu \varepsilon$ in three sections, with the tension length of $0.5 \mathrm{~m}, 1 \mathrm{~m}$, and $1.5 \mathrm{~m}$. As shown in Fig.4, good agreement between the simulation results and the analyzer readings was found, and the correlation coefficient was better than $991 \%$. In addition, both the analyzer measured and the simulated strain shows rather unreliable measurement at sections one and two: comparing to the input strain $(1200 \mu \varepsilon)$, a maximum measurement of $900 \mu \varepsilon$ and $1100 \mu \varepsilon$ are found for sections one and two in the figure. The relatively smaller strain measurement is due to the analyzer's spatial resolution, over which the measured strain is a convoluted result. Therefore, when the strained length of the cable is less than or around the analyzer's spatial resolution, analyzer readings is far smaller than true strain, leading to measurement error.

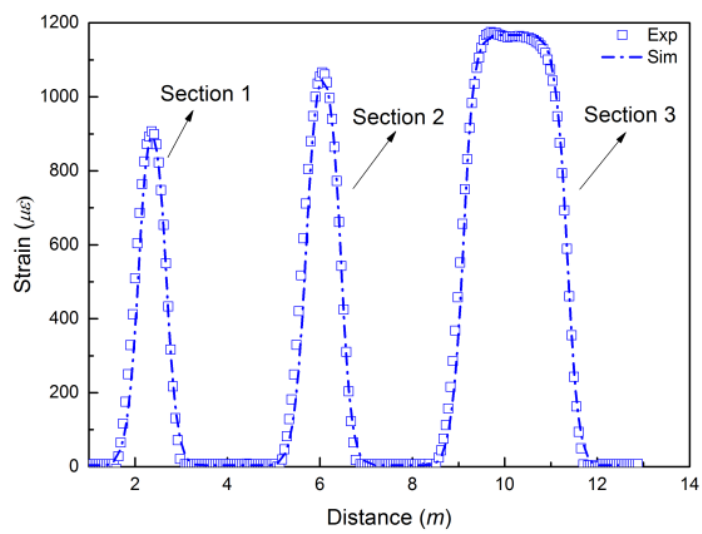

Figure 4. Comparison of the analyzer measured strain and the simulated strain result when the cable is compressed and elongated in different sections. 


\section{PARAMETRIC STUDIES}

Verifying the reliability of the analytical Brillouin scattering simulation method, the constructed model is used to analyze the effects of different factors, including cable parameters (fiber loss and cable length) and analyzer specifications (SNR, frequency scanning step, and spectrum bandwidth, etc.) on the distributed sensor measurement error. The detailed description and graphically illustration of the analyzer parameters are summarized in Fig. 5.

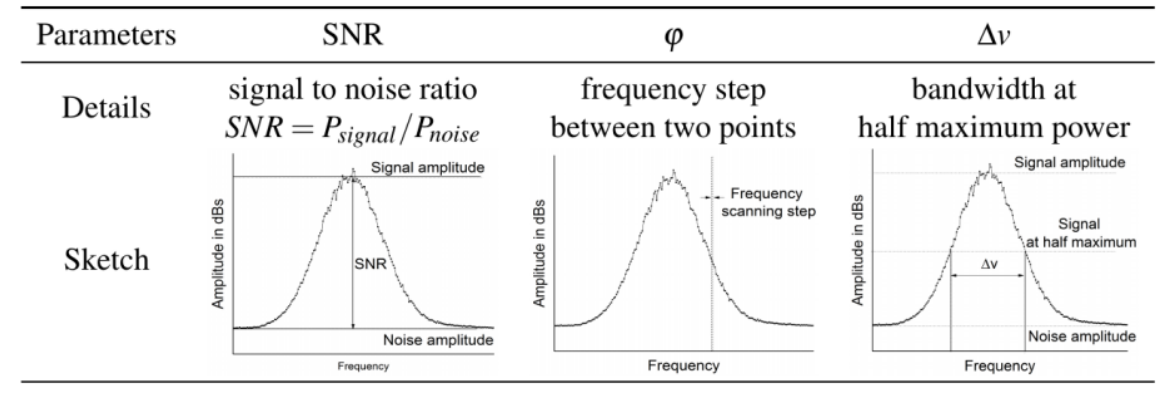

Figure 5. Detailed description and graphically illustration of the analyzer parameters

Cable length and attenuation factor are two parameters that affect the simulation results of the optics model. When light travels through a sensing cable, its power will keep loss through the cable length and thus reduce the SNR of the received measurement. As a result, the measurement error, which is proportionally related to the SNR, is affected by the power attenuation factor of the sensing cable. The influence of the fiber power loss as well as the cable length on the measurement error are therefore investigated. To conduct a parametric study on cable effects, the analyzer inputs are set as constant. For this simulation, the same inputs (pulse shape, power fluctuation characteristics, $3 \mathrm{MHz}$ frequency step and $30 \mathrm{MHz}$ bandwidth) are used.

The simulation is then done with fibre linear loss of $0.25 \mathrm{~dB}$ and $0.23 \mathrm{~dB}$. Figure. 6 compares the measurement error as a function of distance at different fibre attenuation levels. The scattered measurement error is calculated as the standard deviation of the simulated strain obtained in a $200 \mathrm{~m}$ cable section. The lines in the figure represent the respective strain error calculated using a published error calculation equation[11], based on the simulated SNR and bandwidth information.

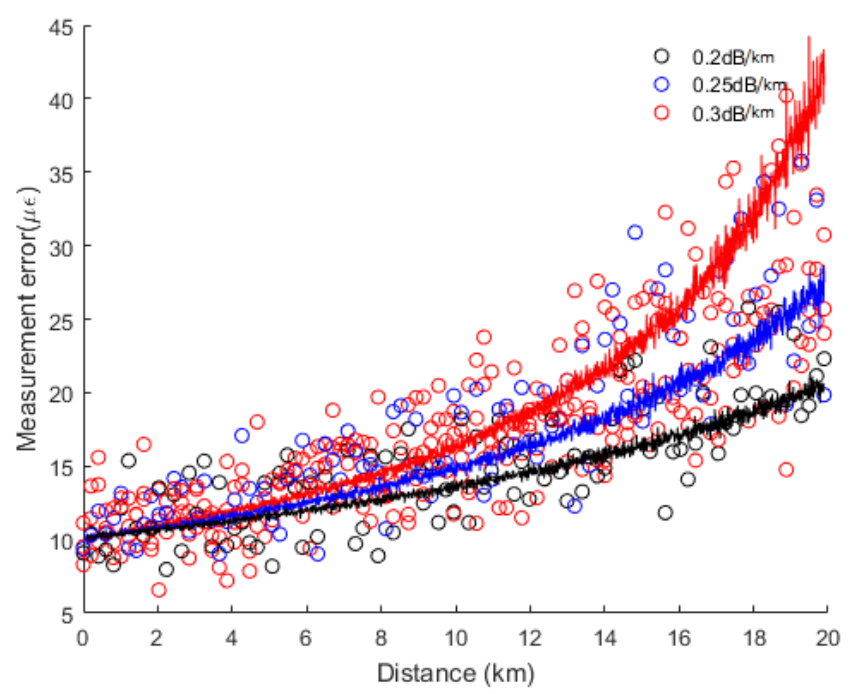

Figure 6. The simulated strain error along the optical fibre of $20 \mathrm{~km}$ length at different attenuation levels

The impact of the fiber attenuation coefficient can be clearly observed in the above figure. When the cable length is shorter than $2 \mathrm{~km}$, the fiber attenuation factor does not have a significant impact on the error. It stays close to $10 \mu \varepsilon$ for all 
three cases. As the location of the measurement gets longer, the error starts to increase with distance. The gradient of the increase is dependent on the attenuation factor, that is, a larger fiber attenuation factor causes a faster error increment along the fiber. The error obtains at $20 \mathrm{~km}$ distance with $0.3 \mathrm{~dB} / \mathrm{km}$ loss is $44.3 \mu \varepsilon$, which is improved down to $21.1 \mu \varepsilon$ with $0.2 \mathrm{~dB} / \mathrm{km}$ loss. This corresponds to an improvement factor of 2.1 on the measurement error by improving fiber loss in $0.1 \mathrm{~dB} / \mathrm{km}$ for $20 \mathrm{~km}$ length of cable.

The analyzer parameters that influence the optics model simulation results are investigated upon a short length of cable to remove any cable effects. The essential discrepancy of the model simulation with the formerly published error equation is checked. The analyzer input parameters includes scanning frequency, SNR, and Brillouin full width at half maximum (FWHM). By changing one parameter and fixing the others, the simulated error dependency on analyzer parameters is obtained and then compared with the equation relations.

The inverse dependency of SNR on error is first investigated. The pulse extinction ratio input of the optics model is used to change the SNR of the received Brillouin signal. Based on typical BOTDR settings, the frequency step is fixed at $3 \mathrm{MHz}$ and the final convoluted Brillouin spectrum FWHM is fixed at $130 \mathrm{MHz}$. Figure 7 illustrates the simulated and calculated results of the error with a changing SNR. An inverse linear relationship between SNR and the random error is obtained from the model, correlating well with the published equation[11].

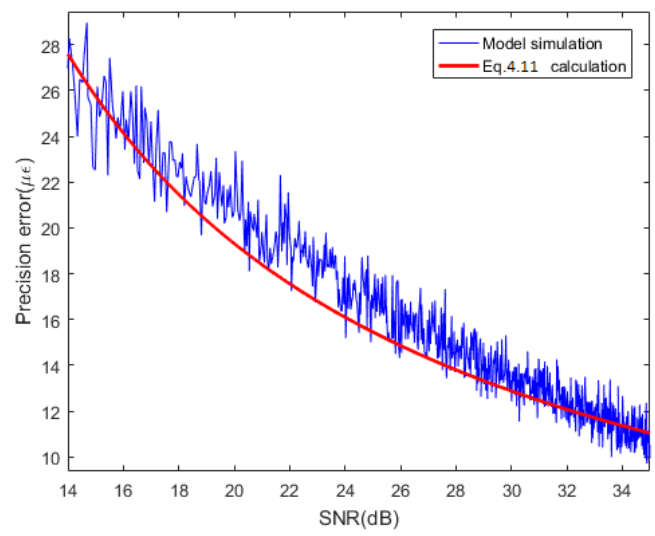

Figure 7. Comparison of the dependence of error on SNR in model simulation and equation calculation results

The dependence of the error on the frequency scan increment is investigated by fixing the SNR and full width at half maximum, and changing the frequency step in the model. Figure 7 shows the simulated error as a function of the frequency scanning step when using 30MHz FWHM and 32dB SNR. It can be observed that a small frequency step (i.e. when more data points are involved in the fitting of the gain spectrum) leads, as expected, to more accurate frequency measurements. Therefore, as shown in Fig.8, if the frequency increment is larger, the frequency error grows with a factor proportional to bandwidth, in full agreement with the published equation. 


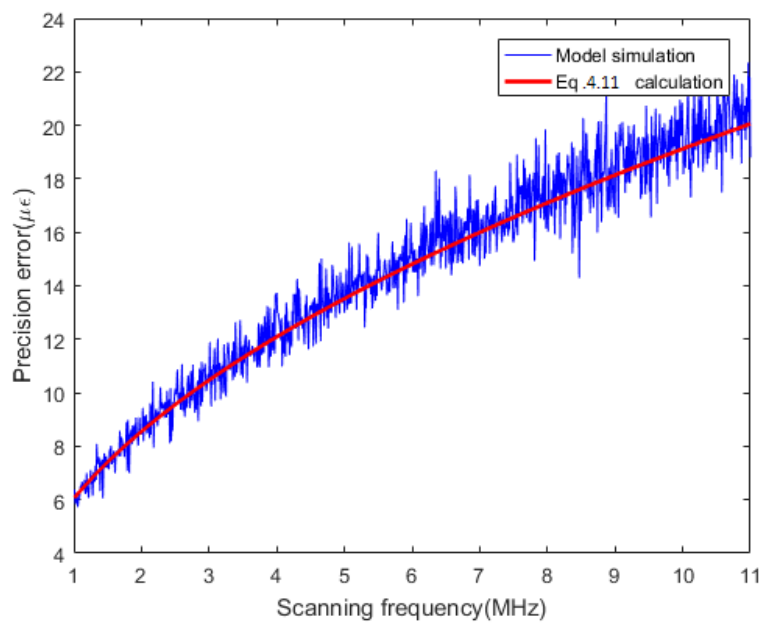

Figure 8. Comparison of the dependence of error on scanning frequency step in model simulation and equation calculation results

Finally, the impact of the Brillouin linewidth on the frequency error is analyzed, as shown in Fig. 9. Here, the Brillouin linewidth is changed using different pump pulse widths (between $10 \$ \mathrm{~ns} \$$ and $50 \$ \mathrm{~ns} \$$ ). However, it is important to take into account when the spatial resolution varies, the local Brillouin gain will change, affecting the SNR of the traces accordingly. To evaluate the impact of the Brillouin spectral width only, the peak pump power is adjusted in order to keep a constant Brillouin gain between measurements. This way, the SNR on the analyzer traces at the peak frequency is the same for all measurements ( $\mathrm{SNR}=32 \mathrm{~dB}$ as in the previous case), making a reliable comparison possible between different Brillouin linewidth conditions.

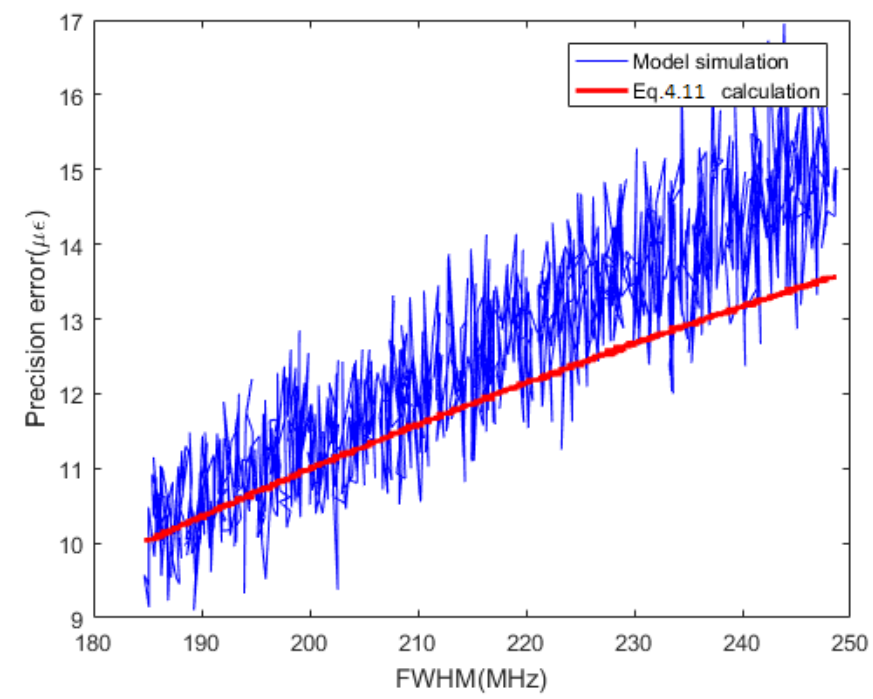

Figure 9. Comparison of the dependence of error on Brillouin linewidth in model simulation and equation calculation results

\section{CONCLUSION}

In this paper, a novel and applicable spontaneous Brillouin backscattering spectrum model is developed. The model includes the injected pulse simulation and the received Brillouin spectrum simulation through a weighted averaging process. The model is experimentally validated with the monitoring cable strained in different lengths and levels. Using this model, parameters that influence optical fiber sensor error is investigated. The impact of the fibre attenuation factor on the error starts to occur when the fiber length is longer than $2 \mathrm{~km}$. There is an improvement factor of 2.1 on the 
measurement error by improving fiber loss by $0.1 \mathrm{~dB} / \mathrm{km}$ for $20 \mathrm{~km}$ length of cable. The error decreases from $28 \mu \varepsilon$ to $11 \mu \varepsilon$ when the SNR increased from $14 \mathrm{~dB}$ to $35 \mathrm{~dB}$. The error increased from 6 to $20 \mu \varepsilon$ when the scanning frequency step increased from $1 \mathrm{MHz}$ to $11 \mathrm{MHz}$. The error increased from $10 \mu \varepsilon$ to $14 \mu \varepsilon$ when the full width at half maximum increased from $185 \mathrm{MHz}$ to $250 \mathrm{MHz}$. With this model, measurement accuracy distributed fiber optics sensor based on spontaneous Brillouin scattering can be better controlled.

\section{REFERENCES}

[1] Li Yongbing and Yang Hong, "Fiber Stress Analyzer," Navigation and Control, vol. 4, pp. 18-18, 2005.

[2] Zhao Lijuan, Li Yongqian, and Xu Zhi Niu, "Lorentzian The influence of the model on the Brillouin spectral feature extraction," external and laser engineering, vol. 5, pp. 218-224, 2016.

[3] M. Bouyahi, A. Zrelli, H. Rezig, and T. Ezzedine, "Modeling the brillouin spectrum by measurement of the distributed strain and temperature," Opt. Quantum Electron., vol. 48, no. 2, pp. 1-10, 2016.

[4] M. Kaur and N. Kaur, "A Novel Approach for Simple Distributed Brillouin Scattering Modeling for Temperature and Strain Sensing Scattering mechanisms :," Int. J. om Recent Innov. trends Comput. Commun., vol. 2, no. 12, pp. 4070-4074, 2014.

[5] D. Garus, T. Gogolla, K. Krebber, and F. Schliep, "Brillouin optical-fiber frequency-domain analysis for distributed temperature and strain measurements," J. Light. Technol., vol. 15, no. 4, pp. 654-662, 1997.

[6] H. Naruse and M. Tateda, "Launched pulse-shape dependence of the power spectrum of the spontaneous Brillouin backscattered light in an optical fiber," Appl. Opt., vol. 39, no. 34, pp. 6376-6384, 2000.

[7] D. Zhang, H. Xu, B. Shi, H. Sui, and G. Wei, "Brillouin power spectrum analysis for partially uniformly strained optical fiber," Opt. Lasers Eng., vol. 47, no. 9, pp. 976-981, 2009.

[8] A. Minardo, R. Bernini, and L. Zeni, "Stimulated Brillouin scattering modeling for highresolution, time-domain distributed sensing.," Opt. Express, vol. 15, no. 16, pp. 10397-10407, 2007.

[9] K. Nishiguchi, C. H. Li, A. Guzik, and K. Kishida, "Synthetic spectrum approach for Brillouin optical time-domain reflectometry," Sensors (Switzerland), vol. 14, no. 3, pp. 47314754, 2014.

[10] H. Naruse, M. Tateda, H. Ohno, and A. Shimada, "Linear strain distribution dependence of the Brillouin gain spectrum," 2002 15th Opt. Fiber Sensors Conf. Tech. Dig. OFS 2002, vol. 41, no. 34, pp. 309-312, 2002.

[11] M. A. Soto and L. Thévenaz, "Modeling and evaluating the performance of Brillouin distributed optical fiber sensors," Opt. Express, vol. 21, no. 25, p. 31347, 2013. 